\title{
Estudio poblacional de Guaiacum sanctum L. (Zygophyllaceae) en los bosques secos de Honduras
}

\author{
Lilian Ferrufino Acosta, Thelma María Mejía Ordoñez ${ }^{1}$ \\ Rafael Enrique Corrales Andino ${ }^{2}$
}

\section{RESUMEN}

Guaiacum sanctum, llamado comúnmente guayacán, se caracteriza por su hábito arbóreo y se distribuye desde la parte central de Suramérica hasta el norte de México, sureste de Estados Unidos y las Antillas Mayores. Actualmente se considera una especie amenazada según la lista roja de la UICN y en el apéndice II de CITES. El objetivo de este estudio fue determinar la distribución y estructura de las poblaciones de guayacán en los remanentes de bosque seco ubicados en los departamentos de Comayagua, El Paraíso, Choluteca, Yoro y Olancho. A partir de la información de herbario y comunicaciones personales, se definieron los sitios de estudio, se delimitaron 18 parcelas y se determinaron tres clases de edades. Se midió la estructura vertical y horizontal y se georreferenciaron los individuos adultos. Los resultados indican que el patrón de distribución espacial es agrupado en todas las categorías y el porcentaje de individuos jóvenes es alta. Se sugiere que esta alta regeneración se debe a que la población se está extendiendo. Los resultados de este estudio serán la base para establecer una estrategia de conservación de esta especie, así como la propuesta de áreas protegidas en el bosque seco en Honduras.

Palabras clave: Guaiacum sanctum, guayacán, distribución espacial, estructura poblacional.

\footnotetext{
Beneficiarias de una sustantiva de la DICYP. Profesoras investigadoras del Departamento de Biología, Facultad de Ciencias, UNAH: lilian.ferrufino@unah.edu.hn

${ }^{2}$ Beneficiario de una beca sustantiva de la DICYP. Profesor investigador del Departamento de Ciencia y Tecnologías de la Información Geográfica, Facultad de Ciencias Espaciales, UNAH: rcorrales@unah.edu.hn
} 


\section{ABSTRACT}

Guaiacum sanctum, commonly called "lignum vitae", is characterized by its arboreal habit and distributed from the central part of South America to northern Mexico, southeastern United States and the Greater Antilles. Now it considered an endangered species according to the IUCN Red List and Appendix II of CITES. The aim of this study was to determine the distribution and structure of population in the remnants of dry forest in the departments of Comayagua, El Paraíso, Choluteca, Yoro and Olancho. From specimens deposited in herbarium and personal communications study sites was defined. 18 plots were demarcated and three age classes are defined. The vertical and horizontal structure was measured and adult individuals were georeferenced. The results indicate that the spatial distribution pattern is grouped in all categories and the percentage of juveniles is high. It is suggested that this regeneration is high because the population is spreading. The results of this study will be the basis for establishing a conservation strategy for this species as well as the proposed protected areas in the dry forest in Honduras.

Keywords: Guaiacum sanctum, guayacan, spatial distribution, population structure. 


\section{INTRODUCCIÓN}

Guaiacum sanctum L. es una especie arbórea de crecimiento lento que pertenece a la familia Zygophyllaceae, habita en el bosque seco subtropical y tropical. Se distribuye desde la parte central de Suramérica hasta el sureste de Estados Unidos y las Antillas Mayores (Stevens y otros, 2001). En Centroamérica es conocida como guayacán, palo santo, guayacán real y lignum vitae en Norteamérica.

La madera tiene importancia económica en la fabricación de pisos de barcos y la resina de los tallos es de uso medicinal, motivo por el cual actualmente se considera una especie amenazada según la lista roja de la UICN y en el apéndice II de CITES. Las poblaciones en México, Centroamérica y Florida disminuyeron en el siglo 19 y se mantienen aisladas debido a la deforestación del bosque, ganadería extensiva y la expansión de la agricultura (Fuchs \& Hamrick, 2010). En Honduras se distribuye en los bosques secos, en el litoral Pacífico y Atlántico del país, y se considera una especie que habita en los bosques más amenazados del neotrópico (Janzen, 1988).

Guaiacum sanctum son árboles con una altura de 20 a $60 \mathrm{~m}$, con una corteza grisácea, rugosa, con fisuras verticales, dura, resinosa y a veces exfoliante en placas. Las hojas son compuestas y opuestas, glabras; hojuelas elípticas, obovadas; posee peciolos glabros, ligeramente pubescentes. Posee flores terminales, solitarias, con un cáliz fusionado, de color morado, sépalos obovados u ovados de $5-7 \mathrm{~mm}$ de longitud; la corola es azul o morada con 5 pétalos; las anteras son más cortas que los filamentos.

Presenta un fruto cápsula, obovado de color amarillo o anaranjado, de $1.5 \mathrm{~cm}$ de longitud aproximadamente; las semillas son elipsoides, negras o café oscuro, con un arilo de color rojo (Standley \& Steyermark, 1946; Stevens y otros, 2001; Cordero \& Boshier, 2003). El polen es dispersado por abejas y avispas a largas distancias (Janzen, 1971). Los frutos son dispersos por las especies de aves: Trogon melanocephalus, Trogon elegans, Tityra semifasciata, Eumomotus momota, Pitangus sulphuratus y Calocitta formosa, las semillas son consumidas por estas aves (Fuchs y otros, 2013; ver figura 1). 


\section{Figura 1. Guaiacum sanctum}
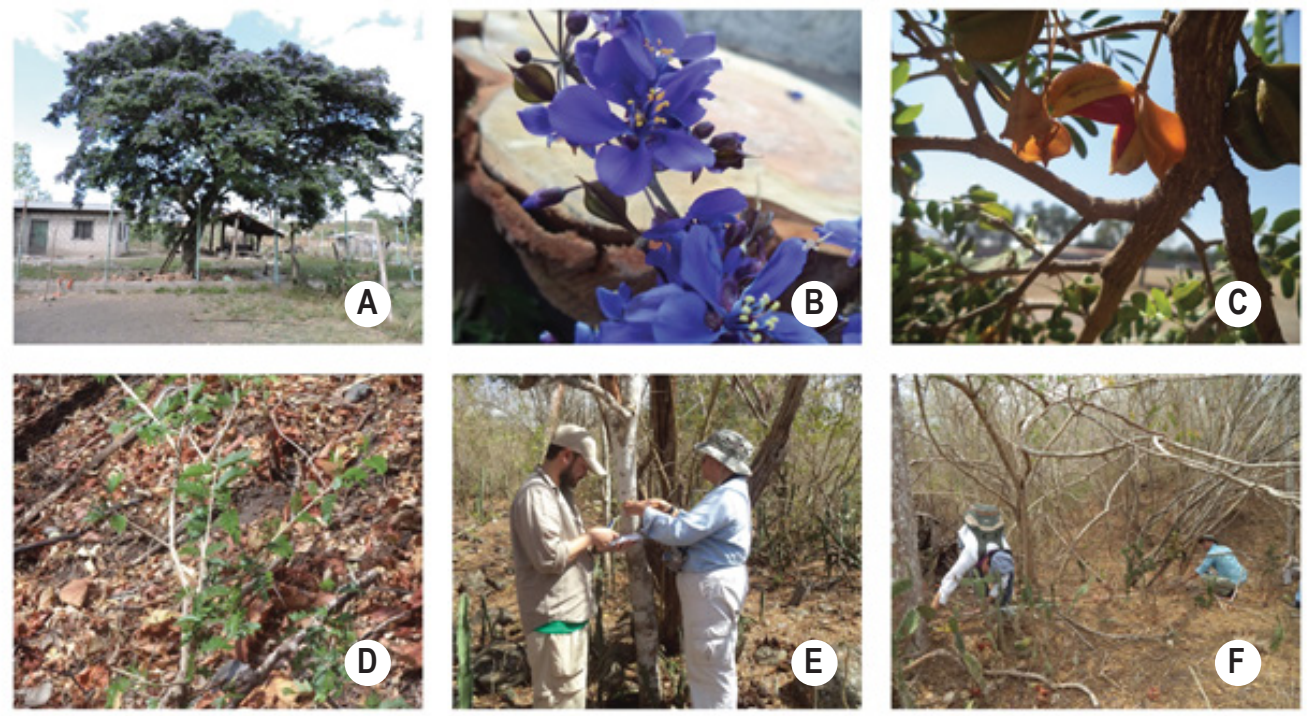

A. flores, B. frutos, C. plántula, D. adulto, E. tomando el DAP a los árboles, F. medición de la distancia entre individuos adultos.

La distribución espacial es una de las propiedades ecológicas más importantes de las especies (ver figura 2). La distribución espacial de los individuos en las poblaciones se ha clasificado en tres patrones básicos: aleatorio, agregado y uniforme (Molles, 2006). En poblaciones naturales de plantas, los individuos suelen distribuirse en forma más o menos agregada, debido a la distribución heterogénea de los recursos, interacción con otras especies, así como por la dispersión restringida de semillas y propágulos vegetativos (Taylor, 1984).

Se han realizados estudios sobre la distribución espacial de G. sanctum en el Parque Nacional Palo Verde en Costa Rica, encontrando altos niveles de diversidad genética. Sin embargo, no se encontró un patrón de estructura genética espacial en la población, lo que sugieren que las semillas están mezcladas y provienen de diferentes adultos (Fuchs y otros, 2010, 2013). También, López Toledo y otros (2011) llevan a cabo un estudio sobre la evaluación del estado de conservación de las poblaciones de G. sanctum y G. coulteri en México, proponiendo un marco de evaluación que se aplique a otras especies amenazadas (López Toledo y otros, 2011).

El objetivo de este estudio fue determinar la distribución y estructura poblacional de $G$. sanctum en los remanentes de bosque seco ubicados en los departamentos de 
Comayagua, El Paraíso, Choluteca, Yoro y Olancho, para conocer el estado de conservación de las poblaciones de esta especie en los bosques secos de Honduras.

\section{MATERIALES Y MÉTODO}

\section{Área de estudio}

El estudio se llevó a cabo en los sitios: 1. Cerro Las Barrancas, El Rodeo y Yuscarán, en el departamento de El Paraíso; 2. El Ojochal y San Marcos de Colón, en el departamento de Choluteca; 3. El Estadio, El Jarín, Las Torrecías y Las Liconas, en el departamento de Comayagua; 4. Arenal, San Lorenzo Abajo y el Refugio de Vida Silvestre Colibrí Esmeralda, en el municipio de Olanchito; 5. Potrero Los Guayacanes y El Potrero de los Hermanos Montoya, en el municipio de Arenal, en el departamento de Yoro; 6. Potrero Don Pedro Carías y El Quebrachal, en el municipio de San Esteban en el departamento de Olancho (ver figura 2). Los sitios fueron seleccionados con base en los especímenes depositados en los herbarios Cyril Hardy Nelson Sutherland (TEFH) y Paul C. Standley (EAP), así como a través de comunicaciones personales de colegas y guías de campo.

\section{Distribución poblacional de Guaiacum sanctum}

\section{Estudio de distribución estructura poblacional de Guaiacum sanctum}

Para la distribución espacial se delimitaron 18 parcelas, de $50 \mathrm{~m} \times 50 \mathrm{~m}$ en áreas disturbadas y fragmentadas y el área protegida Refugio de Vida Silvestre Colibrí Esmeralda. El criterio para la ubicación de las parcelas fue la presencia de un árbol de guayacán adulto, así como de plántulas en regeneración a sus alrededores. Se contó el mayor número de plántulas dentro de la parcela (ver cuadro 1). 
Figura 2. Área de estudio, incluye los sitios de muestreo

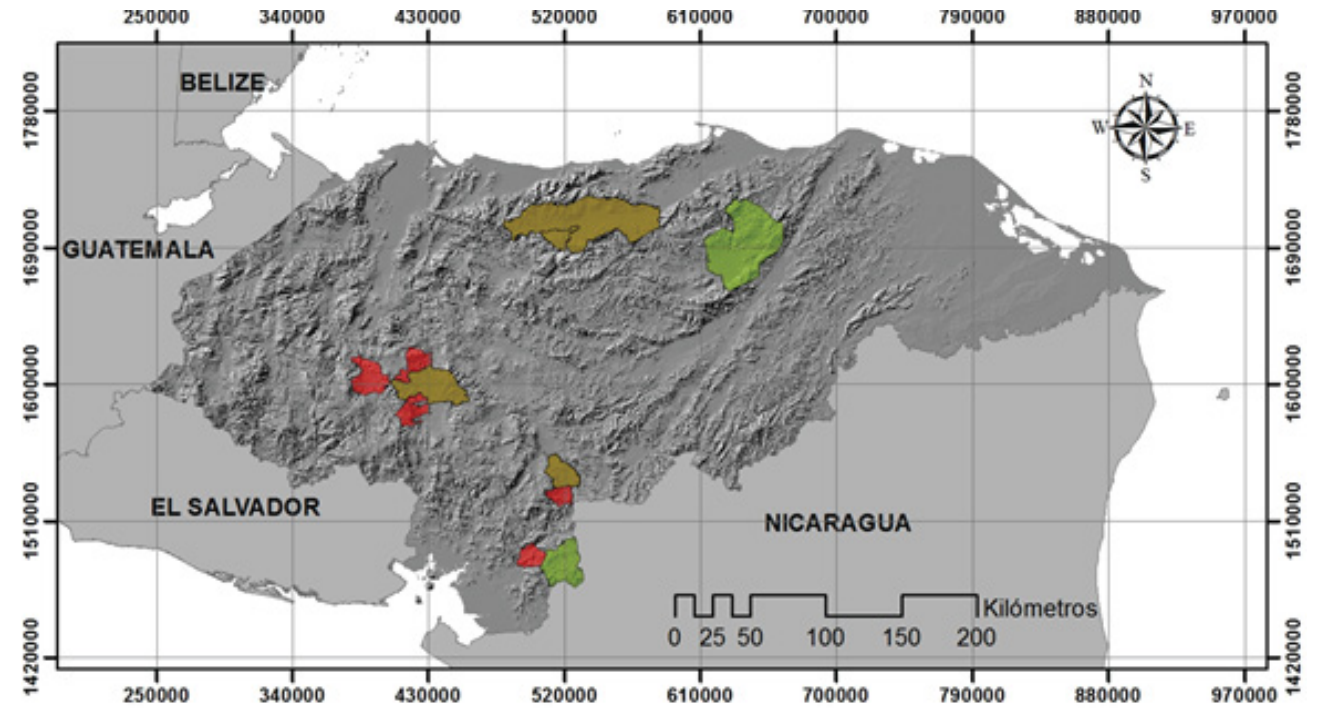

Fuente: propia

\section{Cuadro 1. Sitios y parcelas de muestreos}

\begin{tabular}{|c|c|}
\hline \multicolumn{2}{|c|}{ NOMBRE DEL SITIO / NÚMERO DE PARCELAS } \\
\hline $\begin{array}{l}\text { Sitio1: El Rodeo, Yuscarán, El Paraíso } \\
\text { Parcela 1: Cerro Las Barrancas }\end{array}$ & $\begin{array}{l}\text { Sitio 5: Municipio de Arenal, Yoro } \\
\text { Parcela 11: Potrero Los Guayacanes }\end{array}$ \\
\hline $\begin{array}{l}\text { Sitio 2: Municipio de Comayagua, } \\
\text { Comayagua } \\
\text { Parcela 2: El Estadio } \\
\text { Parcela 3: El Estadio }\end{array}$ & Parcela 12: Potrero de los Hermanos Montoya \\
\hline $\begin{array}{l}\text { Parcela 4: El Jarín } \\
\text { Parcela 5: La Torrecía } \\
\text { Parcela 6: Las Liconas }\end{array}$ & $\begin{array}{l}\text { Sitio 6: El Quebrachal, San Esteban, Olancho } \\
\text { Parcela 13: Potrero de Don Pedro Carías }\end{array}$ \\
\hline $\begin{array}{l}\text { Sitio 3: El Ojochal, San Marcos de } \\
\text { Colón, Choluteca } \\
\text { Parcela } 7 \\
\text { Parcela } 8\end{array}$ & $\begin{array}{l}\text { Parcela 14: Potrero de Don Pedro Carías } \\
\text { Parcela 15: Potrero de Don Pedro Carías } \\
\text { Parcela 16: Potrero de Don Pedro Carías }\end{array}$ \\
\hline $\begin{array}{l}\text { Sitio 4: Municipio de Olanchito, Yoro } \\
\text { Parcela 9: San Lorenzo Abajo } \\
\text { Parcela 10: Refugio de Vida Silvestre } \\
\text { Colibrí Esmeralda }\end{array}$ & $\begin{array}{l}\text { Parcela 17: Potrero de Don Pedro Carías } \\
\text { Parcela 18: Pueblo El Quebrachal }\end{array}$ \\
\hline
\end{tabular}

Fuente: propia 
Los individuos se agruparon en cinco clases de edades según la altura: plántulas $(0-15 \mathrm{~cm})$, retoños (en inglés sampling, $15-30 \mathrm{~cm})$, juveniles $(30 \mathrm{~cm}-2 \mathrm{~m})$, subadultos (2-5 $\mathrm{m}$ ) y adultos ( $\geq 5 \mathrm{~m}$ ), según lo sugerido por Fuchs \& Hamrick (2010) y Fuchs y otros (2013). Se midió el diámetro a la altura del pecho (DAP) y la altura aproximada de cada individuo adulto y la distancia de separación entre ellos (ver figura 2G). También se georreferenció a cada árbol adulto en el campo con un dispositivo de sistemas de geoposicionamiento global (GPS).

\section{Análisis de datos}

Se calculó el índice de Morisita para determinar la distribución espacial de la población por sitio de muestreo, usando la siguiente fórmula:

\section{Donde:}

$q=$ numero parcelas por sitio ni= abundancia por parcela $\mathrm{N}=$ total individuos

$$
\text { Is }=\mathrm{q} \sum_{\mathrm{i}=1}^{\mathrm{q}} \text { ni }\left(\frac{\mathrm{ni}-1}{\mathrm{~N}(\mathrm{~N}-1)}\right)
$$

Además, se calculó la abundancia y densidad total de las poblaciones por sitio y por clases de edad. También se calcularon los promedios de área basal y altura para los adultos. Para estimar la densidad se usó la siguiente fórmula:

Donde:

$\mathrm{S}=$ número de unidades muestrales

$\mathrm{n}_{\mathrm{i}}=$ número de individuos contado en cada unidad

$$
D=\left(s^{-1} \times \sum n_{i}\right) / a
$$

$\mathrm{a}=$ área de cada unidad muestral

\section{RESULTADOS Y DISCUSIÓN}

\section{Distribución espacial de Guaiacum sanctum}

a. Estudio de distribución poblacional de G. sanctum

De acuerdo al índice de Morisita, en las parcelas se observó que los individuos de G. sanctum se distribuyen de manera agregada, como se muestra en la figura 4, para algunos sitios estudiados. Los resultados obtenidos para cada sitio son: 1.03, 1.04, $1.05,1.11,1,53$ y 2.20 , el mayor valor corresponde a Comayagua y el menor valor a la Reserva Biológica Yucarán. 
Se observó que las plántulas y los juveniles muestran un patrón de agregación entre ellos y, a la vez, están muy cercanos a los adultos. Algunos autores comentan que la distribución espacial agregada se debe a dos procesos mutuamente no excluyentes: dispersión de semillas localizadas y la distribución de microhábitat adecuados para la germinación, establecimiento y crecimiento (Forget y otros, 1999; Seidler \& Plotkin, 2006; Fuchs y otros, 2013).

Estos procesos se consideran una ventaja de la distribución espacial agregada, sin embargo, algunos autores comentan que este tipo de distribución conlleva la competencia, lo que provoca una alta mortalidad y reducción de la reproducción sexual (Chadwick, 1991), así como la atracción de los depredadores (Cosner y otros, 1999).

\section{b. Abundancia y densidad de las poblaciones}

Se contaron y midieron 1,630 individuos en 18 parcelas, con un promedio de 90.6 (E.S. 23.97), individuos/parcela. La mayor abundancia se encontró en la comunidad de Comayagua y la menor en la Reserva Biológica Yuscarán (ver figura 3). Se muestreó un total de 4.5 ha y se obtuvo una densidad de individuos con respecto al área muestreada igual a 20.1/ha. La Reserva Biológica Yuscarán tiene la menor densidad $(0.01 / \mathrm{m} 2)$ y la mayor se registró en Comayagua, San Lorenzo Abajo y en el Refugio de Vida Silvestre Colibrí Esmeralda (0.06/m2).

Figura 3. Abundancia de individuos de G. sanctum en el bosque seco de los 6 sitios evaluados

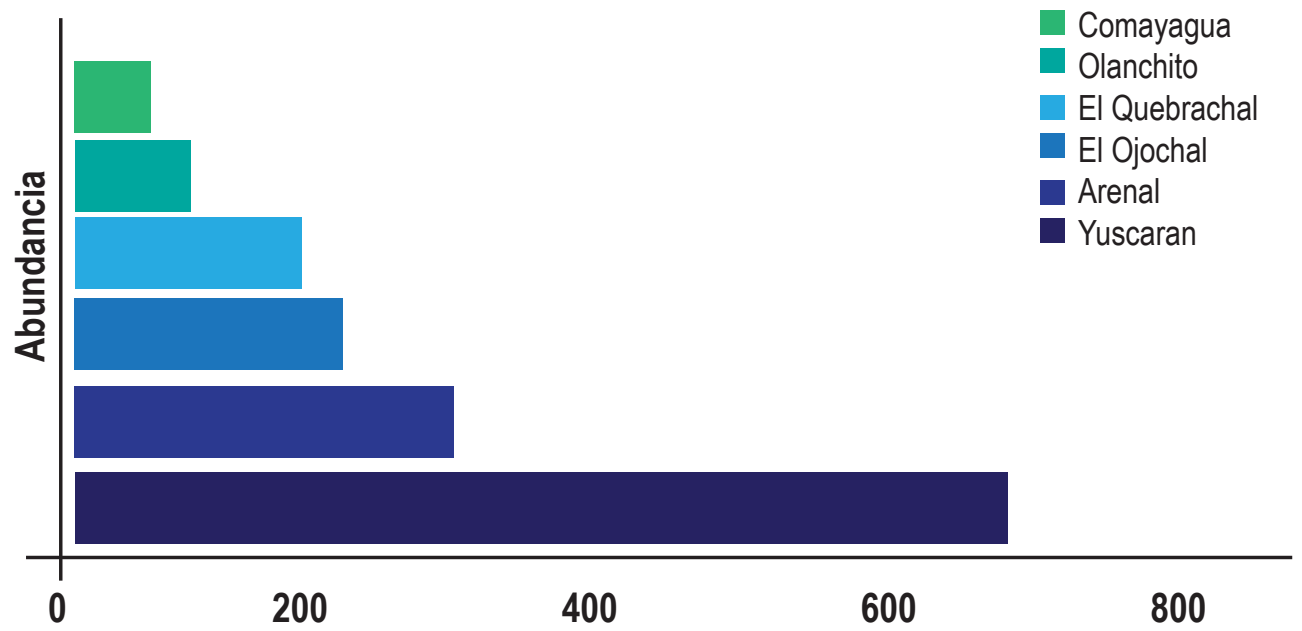

Fuente: propia 


\section{Figura 4. Distribución espacial de los individuos adultos}
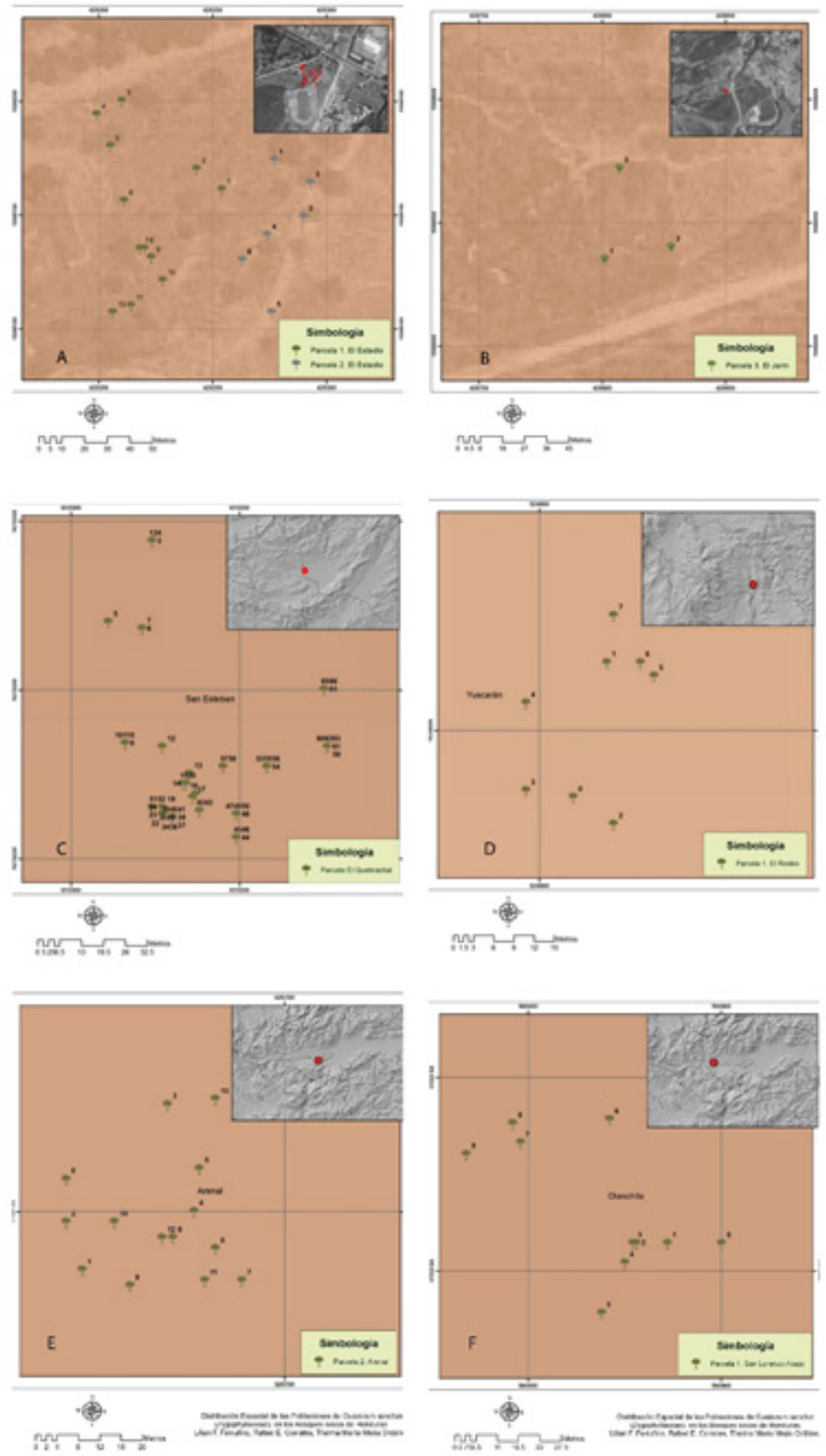

A. flores, B. frutos, C. plántula, D. adulto, E. tomando el DAP a los árboles, F. medición de la distancia entre individuos adultos. 
El conteo y la medición de los individuos se hicieron con base en las clases de edad por tamaño. La categoría más abundante son las plántulas (63\% de los individuos), luego los juveniles (24\%) y finalmente los adultos (13\%), (ver cuadro 2). En Comayagua se registra el mayor número de individuos, en contraste con la Reserva Biológica Yuscarán, en donde se reportan solo ocho individuos (ver figura 4). Fuchs y otros (2013) comentan que la alta densidad de plántulas se debe a que las poblaciones se están extendiendo y que este patrón lo presentan especies longevas con una tasa de crecimiento lento.

\section{Cuadro 2. Abundancia de los individuos de G. sanctum por clases de edad con base en el tamaño}

\begin{tabular}{|l|c|c|c|c|}
\hline \multirow{2}{*}{ Sitios } & Plántulas & Juveniles & Adultos & \multirow{2}{*}{ Total } \\
\cline { 2 - 4 } & de: $0.05 \mathrm{a}<0.50$ & de: $0.50 \mathrm{a}<2.00$ & $\geq 2$ metros & \\
\hline R. B. Yuscarán & 14 & 21 & 1 & 36 \\
\hline Comayagua & 675 & 25 & 26 & 726 \\
\hline $\begin{array}{l}\text { El Ojochal, } \\
\text { Choluteca }\end{array}$ & 53 & 157 & 12 & 222 \\
\hline $\begin{array}{l}\text { R.V. S. Colibrí } \\
\text { Esmeralda, } \\
\text { Olanchito }\end{array}$ & 187 & 48 & 43 & 278 \\
\hline $\begin{array}{l}\text { Arenal, } \\
\text { Olanchito }\end{array}$ & 6 & 38 & 71 & 115 \\
\hline $\begin{array}{l}\text { El Quebrachal, } \\
\text { San Esteban, } \\
\text { Olancho }\end{array}$ & 89 & 109 & 55 & 253 \\
\hline Totales & 1024 & 398 & 208 & 1630 \\
\hline
\end{tabular}

Fuente: propia

La regeneración fue densa alrededor de los árboles madre, estos patrones de distribución se observaron entre los juveniles de mayor tamaño y los adultos (ver figura 5). La herbivoría y los patógenos causan mortalidad de plántulas en algunas especies de árboles neotropicales, aunque se han encontrado la mayoría de los casos en las semillas o plántulas de mortalidad temprana (Augspurger, 1984; Burkey, 1994; Clark y Clark, 1984; Condit y otros, 1992).

Otro posible mecanismo es el dosel, ya que hace claros que permiten la regeneración 
(Augspurger, 1983; Hamill y Wright, 1986; Condit y otros, 1992; Kohyama y otros, 1994). Taylor (1966) comenta que el arilo y la testa inhiben la germinación de las semillas de G. sanctum, sin embargo, bajo condiciones naturales, en particular en la época lluviosa las semillas germinan rápidamente. Por su parte, López Toledo y otros (2011) comentan que la regeneración de G. sanctum es más alta en lugares disturbados, que en zonas menos disturbadas.

\section{Figura 5. Pirámide del tamaño de la población observada de G. sanctum en los sitios muestreados}

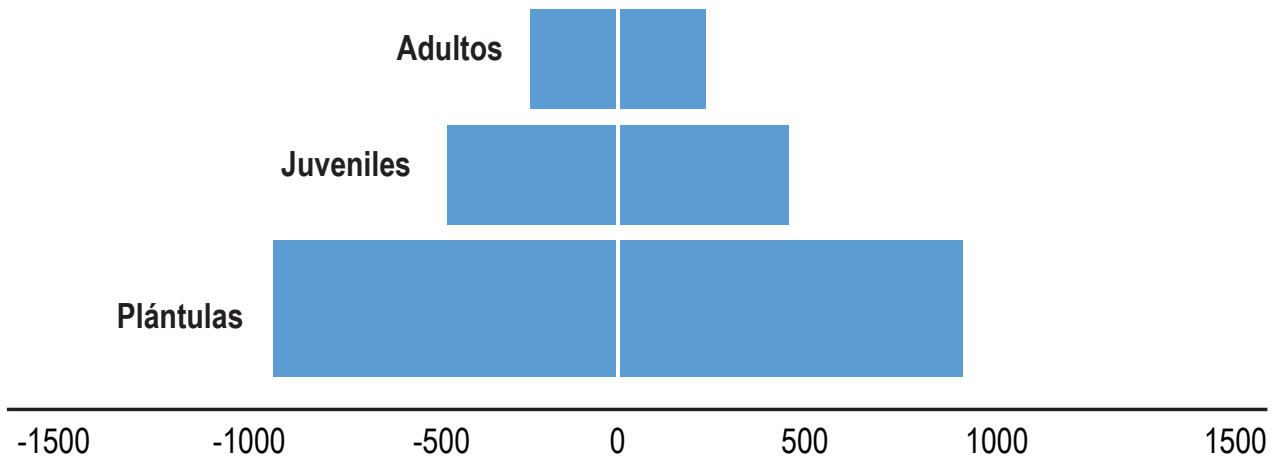

Fuente: propia

La distribución espacialmente de G. sanctum en los bosques secos de Honduras está bajo patrones aleatorios, manteniendo procesos de interacción neutra entre los individuos y su ambiente local, lo que hace que no sea una especie antagónica con otras especies o de atracción de un recurso común, cuando es adulta. Mientras esta especie se encuentra en sus primeros estadios de crecimiento se establece en altas densidades y, por lo tanto, presenta un patrón de distribución espacial agrupada.

\section{c. Estructura de la población}

La estructura vertical de la población arbórea (categoría adultos) del guayacán, se evaluó mediante la altura aproximada de los individuos y la estructura horizontal a partir del DAP (DAP 1.3 m), se midió en esta categoría a todos los individuos mayores de $2 \mathrm{~m}$ de altura. El promedio de altura para todos los arboles resultó igual a $7.6 \mathrm{~m}$, siendo los más altos, con un promedio de $12 \mathrm{~m}$, los que se encontraron en la Reserva Biológica Yucarán y los más pequeños, con promedio de $4.6 \mathrm{~m}$, en Olanchito (ver figura 6). 
Figura 6. Promedio de altura de los individuos de Guaiacum sanctum en cada sitio de muestreo

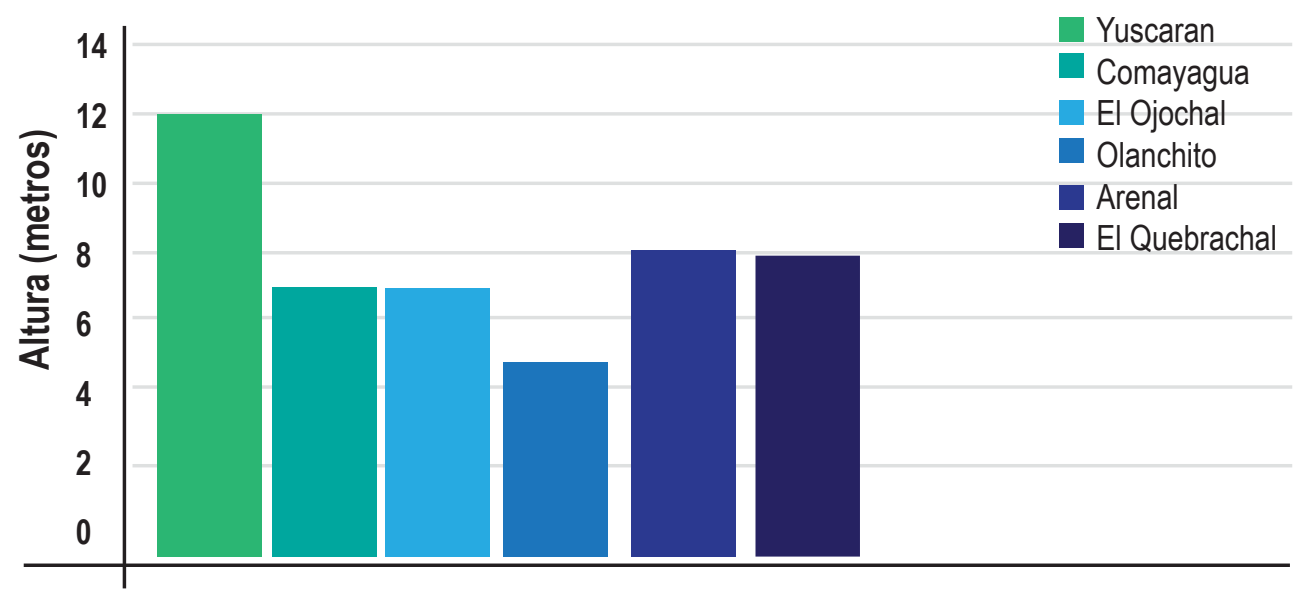

Fuente: propia

La estructura horizontal de los individuos de las parcelas estudiadas mostró que el promedio más alto fue de $32.9 \mathrm{~cm}$, registrado en Comayagua; el menor promedio, $10.6 \mathrm{~cm}$, fue ubicado en Olanchito. El mayor DAP registrado fue de $75 \mathrm{~cm}$ diamétricos para un árbol en Comayagua (ver figura 8), así como el área basal (cobertura de los individuos); se muestra que en Comayagua se obtuvo el valor mayor de $3.40 \mathrm{~cm} 2$ y el menor valor fue de $0.13 \mathrm{~cm}^{2}$ en la Reserva Biológica Yucarán, en este último sitio solo se encontró un árbol con $40 \mathrm{~cm}$ de diámetro (ver figura 7).

Datos promedios de altura y DAP similares se han sido registrados en poblaciones de G. sanctum en Costa Rica (Fuchs y otros, 2013) y México (López Toledo y otros, 2012). La población presenta una distribución diamétrica regular de los árboles censados, como se muestra en otros estudios, lo que se sugiere que es una base para la propuesta de un plan de manejo para la conservación de esta especie en los sitios estudiados (Moret y otros, 2008). Sin embargo, el tamaño promedio de altura y DAP varió entre los sitios. Estas diferencias observadas en las poblaciones de G. sanctum pueden ser una respuesta a las variables climáticas y de suelo (López Toledo y otros, 2012). Otros estudios sugieren que la superficie y la infertilidad del suelo, escasas lluvias y largos periodos de estación seca limitan el desarrollo de esta especie en bosques más densos (White \& Hood, 2004; López Toledo y otros, 2012). 
Figura 7. Promedio de DAP y área basal de la categoría de adultos de G. sanctum por sitios de muestreo

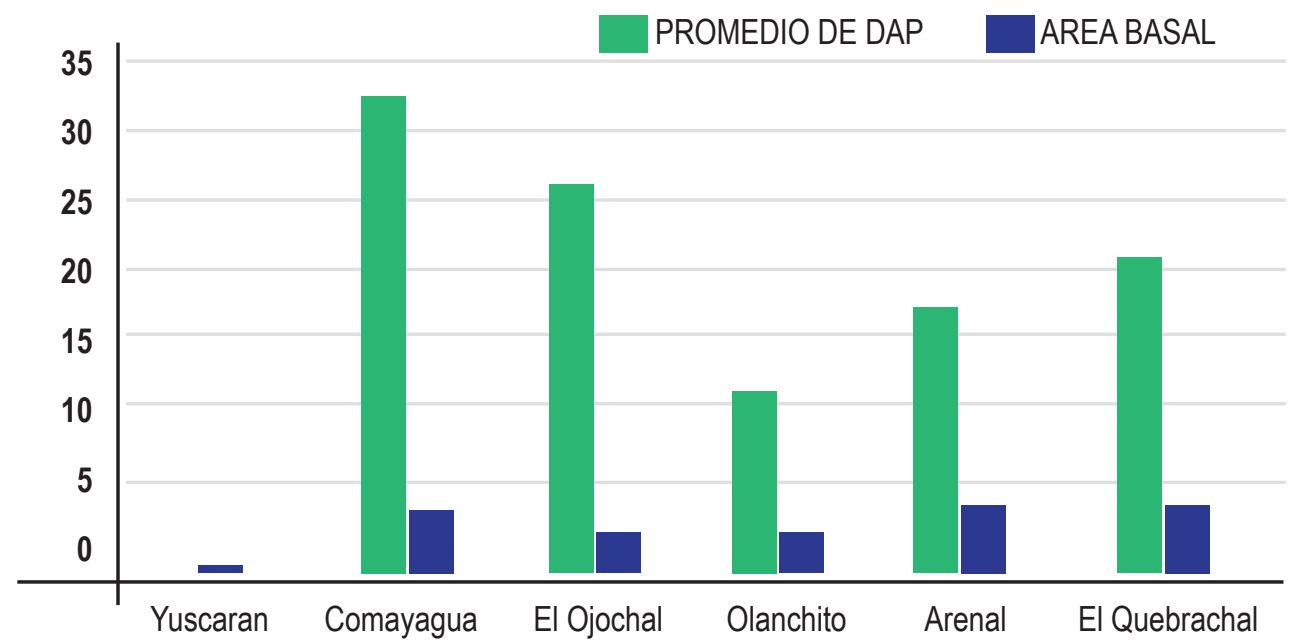

Fuente: propia

\section{CONCLUSIONES}

Una de las principales limitantes ambientales que enfrenta el guayacán es la disponibilidad de agua para los largos periodos de sequía y suelos infértiles. Esto se refleja en el crecimiento lento de la población, registrándose muy pocos individuos de la clase subadultos, en contraste con las plántulas que resultaron muy abundantes.

La distribución espacial de G. sanctum en las poblaciones estudiadas es agregada. Este tipo de distribución la presentan la mayoría de los organismos. Esto se debe a diferentes procesos como la dispersión de las semillas, microclimas que favorecen la germinación, así como al establecimiento y crecimiento de las plántulas. Sin embargo, la deforestación y la falta de incentivos ambientales han conllevado a la falta de conservación de esta especie en áreas no protegidas.

Las poblaciones presentan una alta regeneración debido a los claros que forma el dosel de los árboles adultos; no obstante, herbivoría puede causar la muerte de las plántulas. La mayoría de los sitios muestreados donde habita G. sanctum en Honduras se encuentran en fragmentos de bosques o potreros, López Toledo y otros (2011) sugieren que los sitios disturbados presentan una alta regeneración con relación a los sitios menos disturbados. 
Los resultados de este proyecto serán una herramienta para establecer estrategias de conservación, aprovechamiento de la biodiversidad, estudios de cambio climático, restauración ecológica, así como evaluaciones de servicios ecosistémicos. Además, será útil para la propuesta a CITES y UICN sobre el estatus de conservación de G. sanctum en Honduras.

\section{AGRADECIMIENTOS}

A la Dirección de investigación Científica y Posgrado (DICYP) por el apoyo económico a través de una beca sustantiva. También se agradece la colaboración del Centro Universitario Regional Litoral del Pacífico (CURLP), Centro Universitario Regional del Valle del Aguán (CURVA), Alcaldía de Comayagua y San Marcos de Colón, Fundación Yuscarán, ASIDE y el Herbario TEFH, por facilitar los contactos para la colecta de datos y material vegetal. Gracias a los revisores anónimos.

\section{BIBLIOGRAFÍA}

Augspurger, C. K. (1983). Offspring recruitment around tropical trees: changes in cohort distance with time. Oikos, 40, 189-196.

Augspurger, C. K. \& Kelly, C. K. (1984). Pathogen mortality of tropical tree seedlings: experimental studies of the effects of dispersal distance, seedling density, and light conditions. Oecologia, 61, 211-217.

Burkey, T. V. (1994). Tropical tree species diversity: a test of the Janzen-Connell model. Oecologia, 97, 533-540.

Chadwick, N. E. (1991). Spatial distribution and the effects of competition on some temperate Scleractinia and Corallimorpharia. Mar. Ecol. Prog. Ser., 70, 39-48.

Clark, D. A. \& Clark, D. B. (1984). Spacing dynamics of a tropical rain forest: evolution of the Janzen-Connell model. Am. Nat., 124, 769-788.

Condit, R.; Hubbell, S. P. \& Foster, R. B. (1992). Recruitment near conspecific adults and the maintenance of tree and shrub diversity in a neotropical forest. Am. Nat., 140, 261-286.

Cordero, J. \& Boshier, D.H. (2003). Árboles de Centroamérica. Un manual para extensionista. Costa Rica: CATIE.

Convention on International Trade in Endangered Species of Wild Fauna and Flora (CITES). (s.f.). Apéndices I, II \& III. Recuperado de: https://www.cites.org. 
Cosner, C.; De Angelis, D. L.; Ault, J.S. \& Olson, D. B. (1999). Effects of Spatial Grouping on the Functional Response of Predators. Theoretical Population Biology, 56(1), 65-75. Recuperado de: http://dx.doi.org/10.1006/tpbi.1999.1414

Forget, P. M.; Mercier, F. \& Collinet, F. (1999). Spatial patterns of two rodent-dispersed rain forest trees Carapa procera (Meliaceae) and Vouacapoua americana (Caesalpiniaceae) at Paracou, French Guiana. Journal of Tropical Ecology, 15, 301-313.

Fuchs, E.J. \& Hamrick, J.L. (2010). Genetic Diversity in the Endangered Tropical Tree, Guaiacum sanctum (Zygophyllaceae). Journal of Heredity, 101(3), 284-291.

Fuchs, E.J.; Robles, T. \& Hamrick, J.L. (2013). Spatial distribution of Guaiacum sanctum (Zygophyllaceae) seedlings and saplings relative to canopy cover in Palo Verde National Park, Costa Rica. Rev. Biol. Trop., 61(3), 1521-1533.

Itoh, A.; Yamakura, T.; Ogino, K. \& Lee, H. S. (1995). Survivorship and growth of seedlings of four dipterocarp species in a tropical rain forest of Sarawak, East Malaysia. Ecol. Res., 10, 327-338.

Itoh, A.; Yamakura, T.; Kazuhiko, O.; Lee, H.S. \& Ashton, P.S. (1997). Spatial distribution patterns of two predominant emergent trees in a tropical rainforest in Sarawak, Malaysia. Plant Ecology, 132, 121-136.

Jansen, D.H. (1988). Tropical Dry Forest: the most endangered major tropical ecosystem. In Wilson, E. O. (ed.) Biodiversity. EE.UU.: National Academy Press.

Kohyama, T.; Suzuki, E. \& Hotta, M. (1994). Spatial distribution pattern of representative tree species in a foothill rain forest in West Sumatra. Tropics, 4, 1-15.

Moret, A. Y.; Valera, L.; Mora, A.; Garay, V.; Jerez, M.; Plonczak, M.; Ramírez, N. \& Dimas Hernández, D. (2008). Estructura horizontal y vertical de Pachira quinata (jacq.) W.S. Alverson, (Bombacaceae) en el bosque universitario El Caimital, Barinas, Venezuela. Ecotrópicos, 21(2), 62-74.

Standley, P.C. (1923). Trees and Shrubs of México: Zygophyllaceae, Guaiacum, Contr. U.S. Natl. Herb., 23(3), 522-523.

Stevens, W. D.; Ulloa Ulloa, C.; Pool, A. \& Montiel, O. M. (2001). Flora de Nicaragua. Monogr. Syst. Bot. Missouri Bot. Gard., 85, i-xlii.

Taylor, R.A. (1966). Seed germination and seedling growth of Guaiacum sanctum L. Florida state horticultural society, Vol. 79, 468-470.

Taylor, L.R. (1984). Assessing and interpreting the spatial distributions of insect populations. Ann. Rev. Entomol., 29, 321-357

López Toledo, L.; Ibarra Manríquez, G.; Burslem, D. F.R.P.; Martínez Salas, E.; Pineda García, F. \& Martínez Ramos, M. (2012). Protecting a single endangered species and meeting multiple conservation goals: an approach with Guaiacum sanctum in Yucatan Peninsula, Mexico. Diversity and Distributions, 18, 575-587. Doi:10.1111/j.1472-4642.2011.00857.x 
López Toledo, L.; González Salazar, C.; Burslem, D. F.R.P. \& Martínez Ramos, M. (2011). Conservation Assessment of Guaiacum sanctum and Guaiacum coulteri: Historic Distribution and Future Trends in México. Biotrópica, 43(2), 246-255.

UICN. 2016. The IUCN Red List of Threatened Species. Recuperado de: http://www.iucnredlist.org , consultado en agosto, 2016.

Wehenkel, C.; Brazão Protázio, J.M.; Carrillo Parra, A.; Martínez Guerrero, J.H. \& Crecente Campo, F. (2015). Spatial Distribution Patterns in the Very Rare and Species-Rich Picea chihuahuana Tree Community (Mexico). PLoS One, 10(10), 1-19.

White, D.A. \& Hood, C.S. (2004). Vegetation patterns and environmental gradients in tropical dry forests of the northern Yucatan Peninsula. Journal of Vegetation Science, 15, 151-160. 